University of Wollongong

Research Online

Faculty of Informatics - Papers (Archive)

Faculty of Engineering and Information

Sciences

2007

\title{
Fast RSVP: a cross layer resource reservation scheme for mobile IPv6 networks
}

Yi Sun

Chinese Academy of Sciences

Bin Feng

Chinese Academy of Sciences

Yucheng Zhang

Chinese Academy of Sciences

Gengfa Fang

Chinese Academy of Sciences

Jinglin Shi

Chinese Academy of Sciences

See next page for additional authors

Follow this and additional works at: https://ro.uow.edu.au/infopapers

Part of the Physical Sciences and Mathematics Commons

\section{Recommended Citation}

Sun, Yi; Feng, Bin; Zhang, Yucheng; Fang, Gengfa; Shi, Jinglin; and Dutkiewicz, E.: Fast RSVP: a cross layer resource reservation scheme for mobile IPv6 networks 2007.

https://ro.uow.edu.au/infopapers/3055

Research Online is the open access institutional repository for the University of Wollongong. For further information contact the UOW Library: research-pubs@uow.edu.au 


\title{
Fast RSVP: a cross layer resource reservation scheme for mobile IPv6 networks
}

\author{
Abstract \\ This paper proposes a new cross layer scheme (Fast (Fast RSVP) RSVP) to reserve resources in mobile \\ IPv6 networks. networks. Through the cooperation of mobile IP and RSVP modules, The organ Fast RSVP \\ includes a number of mechanisms such as ad-introduces re vanced resource reservation on neighbor \\ tunnels, resource RSVP schem reservation on optimized routes, resource reservation for results; Secti \\ handover sessions, path merge etc. Network simulation re- conclusions. sults show that our scheme, \\ compared with other traditional ways to reserve resources in mobile environments, has the 2 . Related \\ following advantages: (1) it allows a mobile node to realize fast handover with QoS guarantees; (2) it \\ avoids resource wasting caused by triangular routes and duplicate In recent reservations; (3) it \\ distinguishes different types of reserva- have carried tion requests, greatly reducing the handover session \\ forced vation schem termination rate while maintaining high performance ofthe posals [2-6] network.

\section{Disciplines} \\ Physical Sciences and Mathematics

\section{Publication Details} \\ Yi, S., Feng, B., Zhang, Y., Fang, G., Shi, J. \& Dutkiewicz, E. (2007). Fast rsvp: a cross layer resource \\ reservation scheme for mobile IPv6 networks. IEEE Symposium on Computers and Communications (pp. \\ 691-697). Portugal: IEEE.
}

\section{Authors}

Yi Sun, Bin Feng, Yucheng Zhang, Gengfa Fang, Jinglin Shi, and E. Dutkiewicz 


\section{Fast RSVP: A Cross Layer Resource Reservation Scheme for Mobile IPv6 Networks}

\author{
Yi Sun, Bin Feng, Yucheng Zhang, \\ Gengfa Fang, Jinglin Shi \\ Institute of Computing Technology \\ Chinese Academy of Sciences \\ $\{$ sunyi,fengbin,zhangyucheng,gengfa.fang,sjl\}@ict.ac.cn
}

\author{
Eryk Dutkiewicz \\ University of Wollongong \\ Wollongong, Australia \\ eryk@uow.edu.au
}

\begin{abstract}
This paper proposes a new cross layer scheme (Fast RSVP) to reserve resources in mobile IPv6 networks. Through the cooperation of mobile IP and RSVP modules, Fast RSVP includes a number of mechanisms such as advanced resource reservation on neighbor tunnels, resource reservation on optimized routes, resource reservation for handover sessions, path merge etc. Network simulation results show that our scheme, compared with other traditional ways to reserve resources in mobile environments, has the following advantages: (1) it allows a mobile node to realize fast handover with QoS guarantees; (2) it avoids resource wasting caused by triangular routes and duplicate reservations; (3) it distinguishes different types of reservation requests, greatly reducing the handover session forced termination rate while maintaining high performance of the network.
\end{abstract}

\section{Introduction}

In recent years, deploying multimedia services in next generation mobile IPv6 networks has become an inevitable trend. However, multimedia sessions containing real-time voice and video are very sensitive to delay and delay jitter, and hence have strict QoS requirements. To fulfill the QoS needs of such multimedia sessions, adequate network resources must be reserved for their transmissions. This can be done using the Resource Reservation Protocol (RSVP) [1] that IETF proposed in 1997. However, RSVP is designed for hardwired and fixed networks and when applying it in wireless mobile environments, a lot of problems arise: (1) RSVP control messages cannot be identified in the tunnel; (2) RSVP protocol has no advance resource reservation schemes. (3) RSVP protocol cannot distinguish resource reservation requests from different kinds of ses- sions. Therefore, RSVP is unsuitable for resource reservation in mobile networks. This paper proposes a new scheme (Fast RSVP) to realize resource reservation in mobile IPv6 networks.

The organization of the paper is as follows: Section 2 introduces related research work; Section 3 describes Fast RSVP scheme in details; Section 4 presents the simulation results; Section 5 summarizes the paper and presents our conclusions.

\section{Related Work}

In recent years, experts and scholars all over the world have carried out a lot of research work on resource reservation schemes in mobile environments and a series of proposals [2-6] have been made.

References [2] and [3] proposed a scheme (RSVP Tunnel) to set up RSVP tunnels. The scheme properly solves the problem that the traditional RSVP protocol could not set up resource reservation in tunnels, but it has the following weaknesses: (1) It has no advance resource reservation mechanism. In addition, the setup of resource reservation in the tunnel is triggered by the end-to-end RSVP refresh messages after a mobile node hands over to the new subnet. Therefore, the QoS of session cannot be guaranteed during the time that the mobile node hands over to the new subnet but has not finished the resource reservation process in the tunnel. (2) The scheme utilizes a resource reservation tunnel between the Home Agent and Foreign Agent to transmit session data, but has no route optimization consideration. Thus resource waste is inevitable because of the triangular route. (3) The scheme does not distinguish between reservation requests from new sessions and those from handover sessions.

MRSVP scheme [4] and Multicast RSVP scheme [5] support advance resource reservation in handover target subnet, thus realizing mobile node handover with QoS guar- 
antees. However, these schemes have the following weaknesses: (1) They require passive resource reservations in all neighbor subnets for a mobile node which may lead to significant resource wasting. (2) When a mobile node hands over to a new subnet, all the resources reserved for it on the old path become useless, and the schemes do not consider taking full advantage of the resources already reserved on the old path. (3) The schemes do not distinguish between reservation requests from new sessions and those from handover sessions.

Reference [6] proposed a link layer assisted multicastbased reservation scheme (LM-MRSVP) utilizing $802.11 \mathrm{~b}$ link layer information to make handover predictions. This link layer assisted reservation scheme would effectively avoid the over-reservation problem. However, it has the following disadvantages: (1) When a mobile node hands over to a new subnet, all the resources reserved for it on the old path become useless, and the scheme does not consider taking full advantage of the resources already reserved on the old path. (2) Because the scheme is based on multicast technology, when handover prediction fails, the mobile node has to rebuild a totally new multi-hop reservation path. Thus the reservation setup time is relatively long and during this period of time the QoS of the session cannot be guaranteed. (3) The scheme does not distinguish between reservation requests from new sessions and those from handover sessions.

\section{Fast RSVP}

In the Fast RSVP scheme, a handover process with QoS guarantees could be divided into 2 stages: (1) setup of the resource reservation neighbor tunnel and (2) resource reservation on the optimized route. As illustrated in Fig. 1, after the mobile node (MN) moves to a new subnet, it first communicates with the corresponding node $(\mathrm{CN})$ utilizing the resource reservation tunnel which is set up ahead of the handover event (illustrated by the solid line in Fig. 1). When $\mathrm{MN}$ gets stable in the new subnet, it starts the resource reservation process on the optimized route. After resources are successfully reserved on the optimized route, the sessions between $\mathrm{MN}$ and $\mathrm{CN}$ are smoothly switched from the reservation tunnel to the new optimized route (illustrated by the dashed line in Fig. 1).

\subsection{Resource Reservation on Neighbor Tunnel}

In order to better mark the multimedia sessions in mobile environments, Fast RSVP imports a new object MSESSION to replace the SESSION object in the traditional RSVP protocol. Compared with the SESSION object, MSESSION

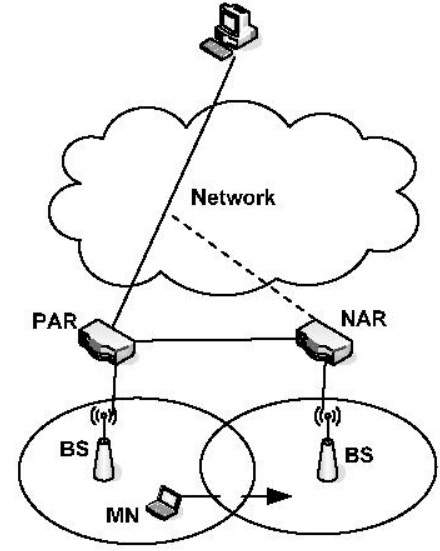

Figure 1: Example of Fast RSVP. The solid line depicts the resource reservation tunnel and the dashed line represents resource reservation on optimized route after handover.

adds a "home address" field in the object. In mobile environments, the RSVP router should label a multimedia session based on the content of MSESSION and set up the corresponding Path State and Resv State accordingly. Fast RSVP relies on the fact that a mobile node can predict its handover event. This requirement is not hard to be met, and a lot of handover prediction algorithms $[7,8]$ can be used here.

When the mobile IP module of MN predicts that a handover event from PAR (Previous Access Router) to NAR (New Access Router) is imminent, it sends an indication message HandoverForecast.ind to RSVP module and tells the RSVP module the new care-of address that will be used by MN in NAR's subnet. The RSVP module utilizes the new care-of address to form the MSESSION object and sends a HandoverForecast message (containing MSESSION) to the current access router PAR. When PAR receives the HandoverForecast message, it looks up the matching Path State of the session according to the "Home Address", "Dest Port" and "Protocol Id" in the MSESSION object. Then the RSVP module of PAR extracts the SENDER_TSPEC (defines the traffic characteristics of a sender's data flow) [1] contained in the matching Path State, constructs the TunnelPath message accordingly, and sends the message to NAR. The TunnelPath message includes the SENDER_TSPEC and MN's new care-of address which can be found in the HandoverForecast message.

When intermediate routers receive the TunnelPath message, they set up the Path State for the session according to the content of MSESSION and forward the TunnelPath message to the next hop. Finally, when the router of the handover target subnet (NAR) receives the TunnelPath message, it makes advance resource reservation for the session locally and sets up the corresponding Path State, Resv State. After that, NAR replies with a TunnelResv message. 
When intermediate routers receive the TunnelResv message, they make advance resource reservation for the session locally, set up the corresponding Resv State and forward the TunnelResv message to the previous hop. Finally, when PAR receives the TunnelResv message, advance resource reservation on the neighbor tunnel between PAR and NAR successfully completes.

The TunnelPath and TunnelResv messages share the same format with the Path and Resv messages, except that they have a different "Msg Type" field value in Common Header to indicate that they correspond to tunnel resource reservation.

After MN hands over to a new subnet, its mobile IP module sends a BU message to PAR, so that PAR can redirect the session data to MN's new position. Besides, the mobile IP module of MN should check whether the current subnet is the same as the predicted handover target subnet. If it is the same, $\mathrm{MN}$ and $\mathrm{CN}$ can communicate with each other through the resource reservation neighbor tunnel and the QoS of the communication can be guaranteed. Otherwise, the handover prediction is wrong and then the mobile IP module of MN should send an indication message HandoverForecastError.ind to the RSVP module. This indication message contains the current in use care-of address and the formerly falsely predicted care-of address of MN. When the RSVP module of MN receives this indication message, it sends a HandoverForecastError message to PAR. After receiving the HandoverForecastError message, the RSVP module of PAR extracts MN's current care-of address and formerly falsely predicted care-of address from the payload of the message. Based on this address information, PAR sends a TunnelPathTear message to release the previously mistakenly built reservation tunnel and sends a new TunnelPath message to rebuild the resource reservation tunnel with MN's current subnet. If the handover event does not occur within a given time after MN made a handover prediction, the mobile IP module of $\mathrm{MN}$ should also send a HandoverForecastError.ind message to notify RSVP module that the handover prediction fails, so that RSVP module can send a HandoverForecastError message to PAR to release the resources reserved in advance on the falsely built tunnel.

\subsection{Resource Reservation on Optimized Route}

The use of tunnels induces the triangular route problem, thus causing unnecessary network resource waste. Thus, Fast RSVP should consider how to reserve resources for multimedia sessions on the optimized route. By adding a new cache (Pending Cache) maintained by the mobile IP module and utilizing several indication messages between the RSVP module and the mobile IP module, Fast RSVP scheme can realize resource reservation on the optimized route and ensure that the session data are switched from the old path (neighbor tunnel) to the new optimized route seamlessly.

When MN gets stable in the handover target subnet, it sends a BU message to $\mathrm{CN}$. After the mobile IP module of $\mathrm{CN}$ receives this $\mathrm{BU}$ message, it updates the corresponding entry of $\mathrm{MN}$ in its Pending Cache to record the $<$ home address, care-of-address $>$ pair, and sends a SessionHandover.req message to the RSVP module inquiring whether or not to redirect application data to the new optimized route. On receiving the SessionHandover.req message, the RSVP module of CN checks whether the mobile node (indicated in the SessionHandover.req message) has multimedia sessions requiring resource reservation. If the mobile node (MN) has no such sessions, the RSVP module immediately sends a SessionHandover.ind message to the mobile IP module so that all the packets from $\mathrm{CN}$ to $\mathrm{MN}$ without QoS requirements are switched to the optimized route at once. If $\mathrm{MN}$ does have sessions with resource reservation requirements, the RSVP module of $\mathrm{CN}$ must start the resource reservation process on the optimized route first before it sends a SessionHandover.ind message to the mobile IP module.

The RSVP module of $\mathrm{CN}$ initiates the resource reservation process on the optimized route by sending a Path message to MN. It extracts the new care-of address and home address of $\mathrm{MN}$ from the SessionHandover.req message and generates the MSESSION object accordingly. Then the RSVP module of $\mathrm{CN}$ encapsulates the Path message and sends it to MN.

After an intermediate RSVP router on the transmission path receives the Path message, it checks whether it already has the corresponding Path State of the session according to the "Home Address" "Dest Port" and "Protocol Id" in the MSESSION object. If the corresponding Path State is found, the RSVP module on the router only needs to update this state; otherwise, it sets up the corresponding Path State for the session. In addition, the RSVP module on the router should notify the mobile IP module of MN's home address so that the mobile IP module could fill this address in "Type 2 Routing Header" [9] when re-encapsulating the Path message.

After the Path message corresponding to the optimized route finally reaches $\mathrm{MN}$, the RSVP module of $\mathrm{MN}$ replies with the proper Resv message. After an intermediate RSVP router on the transmission path receives the Resv message, it checks whether it already has the corresponding Resv State of the session also according to the "Home Address" "Dest Port" and "Protocol Id" in the MSESSION object. If the corresponding Resv State is found, the RSVP module on the router only needs to update this state; otherwise, it tries to reserve resources for the session locally and set up 
the corresponding Resv State.

Finally, when the RSVP module of $\mathrm{CN}$ receives the Resv message, the resource reservation process on the optimized route successfully completes. Then the RSVP module sends an indication message SessionHandover.ind to the mobile IP module. On receiving this indication, the mobile IP module of CN updates the proper entry in the Bindng Cache, thus the session data is redirected to the optimized route from then on.

\subsection{Distinguishing Resource Reservation Requests from Different Kinds of Ses- sions}

Experience shows that users are more sensitive to terminating an ongoing session compared with blocking a new session. So Fast RSVP introduces a new mechanism to distinguish resource reservation requests from different kinds of sessions, giving handover sessions a higher priority, therefore reducing the forced termination rate of sessions due to handover.

In Fast RSVP, we stipulate that each router reserves a given amount of its resources only to support handover sessions. Suppose that the total amount of resources that a router has is $\mathrm{C}$, the amount of resources specially reserved for handover sessions is $K(K \leq C)$ and the amount of resources already in use is $N(N \leq C)$.

When the router receives a reservation request (the amount of resources in request is $q$ ), it checks that the request is included in a Resv message or a TunnelResv message. If the request is included in a Resv message, it means that this request is either from a new session or corresponding to resource reservation on the optimized route. For this kind of request, the router would compare $N+q$ and $C-K$. If the former is not bigger than the latter, the request is admitted, otherwise it is rejected. If the request is included in a TunnelResv message, it means that this request is from a handover session. For this kind of request, the router compares $N+q$ and $C$. If the former is not bigger than the latter, the request is admitted; otherwise it is rejected.

Network administrators should establish the exact amount of resource reserved for handover sessions according to the distribution of different types of sessions in the network.

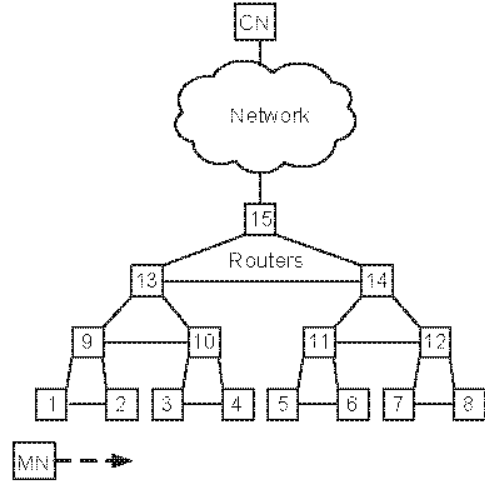

Figure 2: Simulation scenario

\section{Simulation Results}

\subsection{Impact of Utilization of Advanced Re- source Reservation Neighbor Tunnel on the QoS of Sessions during Han- dover}

The simulation scenario is shown in Fig. 2. Initially, MN stays in the subnet of Router 1 and starts a voice session with $\mathrm{CN}$ when simulation time reaches $120 \mathrm{~s}$. Then MN moves towards Router 2 and handover occurs at about $240 \mathrm{~s}$.

Fig. 3 and Fig. 4 respectively depict the end-to-end voice packet delay for the RSVP Tunnel $[2,3]$ and for our Fast RSVP scheme. It can be seen that in Fig. 3 packets have very long delays during 240 to 270 s (the longest delay exceeds $3 \mathrm{~s}$ ). This is because in the RSVP Tunnel scheme, the setup of resource reservation tunnel is triggered by the event that $\mathrm{MN}$ receives a refresh Path message from $\mathrm{CN}$ after handing over to the new subnet. The default value of the Path message refresh period in RSVP Tunnel scheme is $30 \mathrm{~s}$, so during this period the QoS of the session cannot be guaranteed due to the lack of resource reservation in the tunnel. However, in Fast RSVP, because we already reserve adequate resources in the tunnel for $\mathrm{MN}$ before it hands over to the target subnet, the QoS of the multimedia sessions on $\mathrm{MN}$ can be guaranteed (the end-to-end packet delay is restricted to $0.3 \mathrm{~s}$ ).

\subsection{Impact of Different Tunnel Policies on Network Performance}

In this subsection, we simulated and analyzed the impact of different tunnel policies (no tunnel [4-6], home tunnel $[2,3]$ neighbor tunnel in our scheme) on the network performance indicators such as new session blocking rate, handover session forced termination rate, overall session completion rate etc. The simulation parameters 


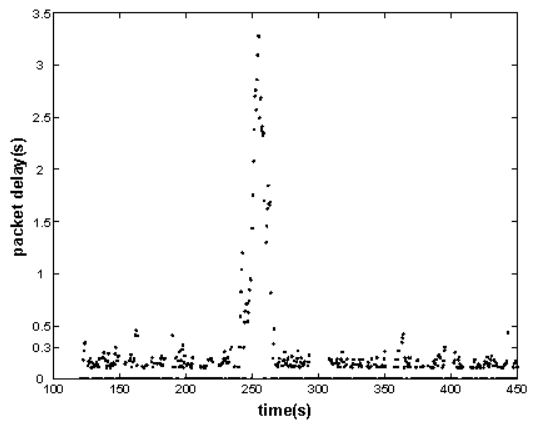

Figure 3: End-to-end voice packet delay in RSVP Tunnel

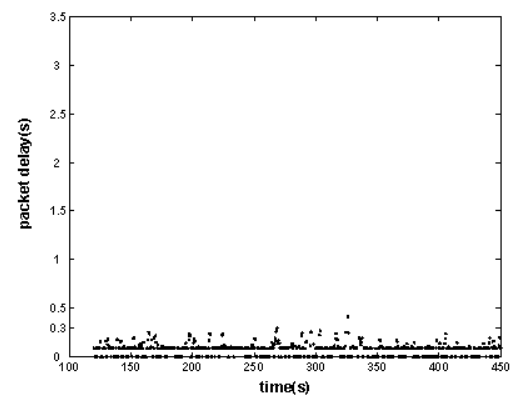

Figure 4: End-to-end voice packet delay in Fast RSVP

are as follows: the arrival of new sessions and handover events are both Poisson distributed and the corresponding parameters are $\lambda_{S}$ and $\lambda_{h}$ respectively. The holding time of new sessions and handover events are both exponentially distributed, with the corresponding parameters $1 / \mu_{S}$ and $1 / \mu_{h}$. The system capacity is $\mathrm{C}$ and the amount of resources that every session needs is $r$ (evenly distributed between $r_{\min }$ and $r_{\max }$ ). The system payload intensity is defined as $\rho=\left(\lambda_{S} \cdot r\right) /\left(\mu_{S} \cdot C\right)$. We define tunnel proportion as the ratio of tunnel length to whole path length after handover and its value depends on the specific tunnel policy. We define new session blocking rate $P_{b}$ as the probability that new sessions are blocked due to lack of system resources, and handover session forced termination rate $P_{f}$ as the probability that sessions are rejected during handover due to lack of system resources. Overall session completion rate $P_{c}$ is the probability that sessions successfully initiate without forced terminating during handovers and finally smoothly complete. The exact values of the above parameters are: $\lambda_{S}=10100 \mathrm{sessions} / \mathrm{sec}, \mu_{S}=$ $0.01 \mathrm{sec}^{-1}, C=10 \mathrm{Mbps}, r_{\min }=64 \mathrm{Kbps}, r_{\max }=$ $2 \mathrm{Mbps}, \lambda_{h}=100$ events $/ \mathrm{sec}, \mu_{h}=0.1 \mathrm{sec}^{-1}$. By changing $\lambda_{S}$, we adjust the system payload intensity $\rho$, and observe the variations of $P_{b}, P_{f}, P_{c}$ when applying no tunnel, home tunnel and neighbor tunnel policies during handover.

The simulation time is 1day and the results are shown in

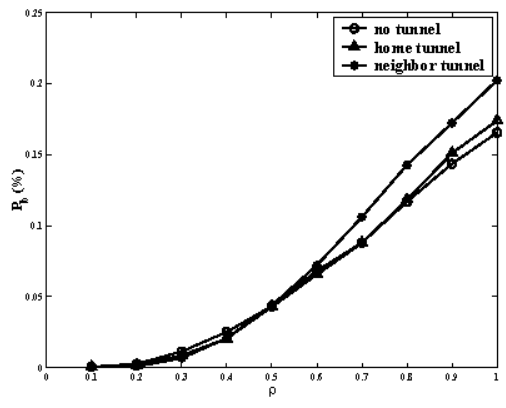

Figure 5: The variation of new session blocking rate $P_{b}$ as the system payload intensity $\rho$ changes using different tunnel policies.

Fig. 5 to Fig. 7. It can be seen that $P_{b}$ and $P_{f}$ both increase as the system payload intensity goes up. Utilization of the tunnel causes a slight rise in new session blocking rate $P_{b}$ (home tunnel $2 \%$, neighbor tunnel $5 \%$ ), but greatly reduces the forced termination rate of handover sessions (home tunnel about $4 \%$, neighbor tunnel about $10 \%$ ). This is because when using the tunnel, the mobile session can make full use of the resources already reserved on the old path and need not set up resource reservations on a totally new path, so the forced termination rate due to handover can be remarkably reduced. However, since more system resources are occupied by handover sessions, the new call blocking rate might increase when the system payload is heavy. The neighbor tunnel policy used in Fast RSVP has a larger ratio of reuse of the already reserved resources compared with the home tunnel policy and fewer new resources are needed when reserving on the neighbor tunnel. Thus the improvement of handover session forced termination rate $P_{f}$ in our scheme is more significant. Also as can be seen from Fig. 7, the overall session completion rate $P_{c}$ declines as the system payload intensity increases. Use of the tunnel improves $P_{c}$, because the overall session completion rate $P_{c}$ is related to the new session blocking rate $P_{b}$ as well as handover session forced termination rate $P_{f}$. The tunnel policy greatly reduces $P_{f}$ and only brings a slight rise of $P_{b}$ when the system payload is heavy, therefore achieving the improvement in $P_{c}$.

\subsection{Impact of Path Merge Mechanism on Network Performance}

In Fast RSVP we make use of a new mechanism named Path Merge. The mechanism utilizes Home Address in the MSESSION object to label a mobile node so that when doing reservation on the optimized route, duplicate reservations are avoided on the intermediate nodes and links shared by the old and new routes. Thereby, Path Merge is expected to improve the network performance.

We define a new simulation parameter Path Merge Ra- 


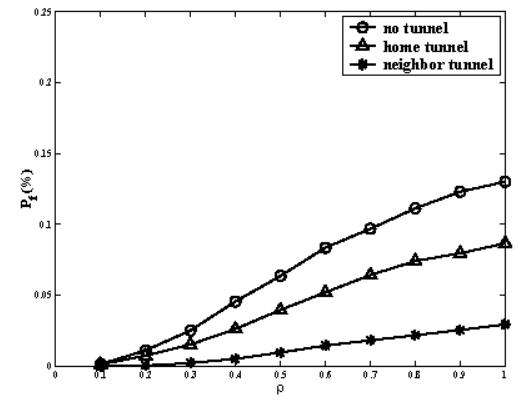

Figure 6: The variation of handover session forced termination rate $P_{f}$ as the system payload intensity $\rho$ changes using different tunnel policies.

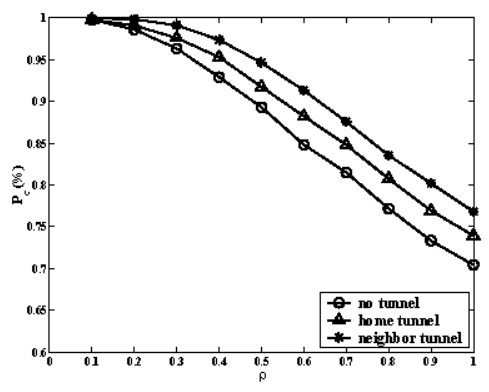

Figure 7: The variation of overall session completion rate $P_{c}$ as the system payload intensity $\rho$ changes using different tunnel policies.

tio $\eta$ as the proportion of the shared route length to the whole route length. The exact values of the simulation parameters are: $\lambda_{S}=10100$ sessions $/ \mathrm{sec}, \mu_{S}=$ $0.01 \mathrm{sec}^{-1}, C=10 \mathrm{Mbps}, r_{\min }=64 \mathrm{Kbps}, r_{\max }=$ $2 \mathrm{Mbps}, \lambda_{h}=100$ events $/ \mathrm{sec}, \mu_{h}=0.1 \mathrm{sec}^{-1}, \phi=$ 0.2 (neighbortunnel), $\eta=0.6$. By changing $\lambda_{S}$, we adjust the system payload intensity $\rho$. The variations of new session blocking rate $P_{b}$, handover session forced termination rate $P_{f}$, overall session completion rate $P_{c}$ and optimized route reservation successful rate $P_{o}$ are shown in Fig. 8 to Fig. 11 respectively. (The simulation time is 1 day.)

As can be seen from Fig. 11, Path Merge remarkably en-

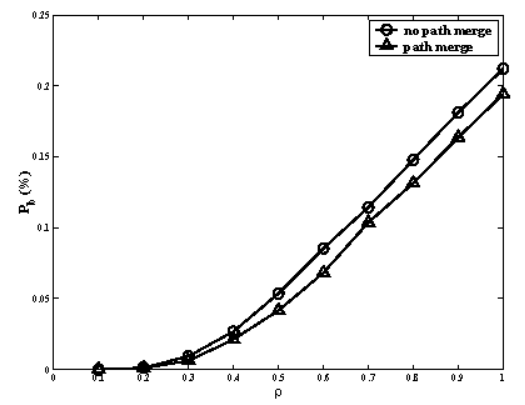

Figure 8: Impact of Path Merge mechanism on the new session blocking rate $P_{b}$.

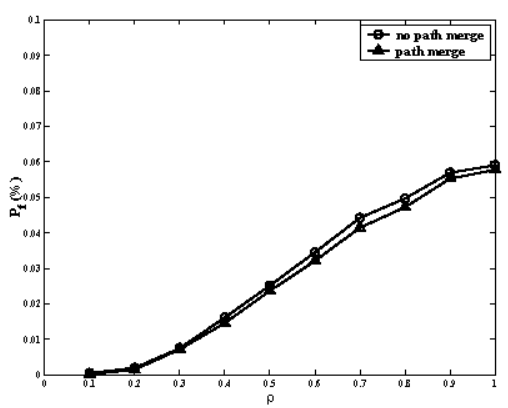

Figure 9: Impact of Path Merge mechanism on the handover session forced termination rate $P_{f}$.

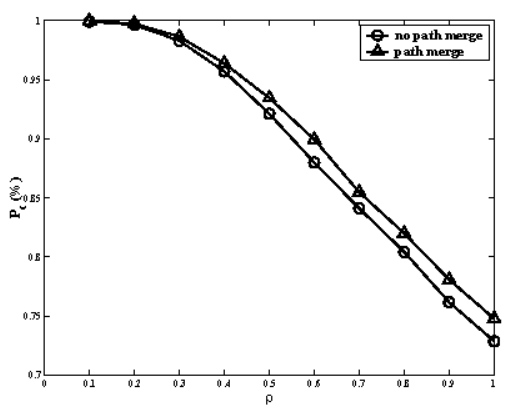

Figure 10: Impact of Path Merge mechanism on the overall session completion rate $P_{c}$.

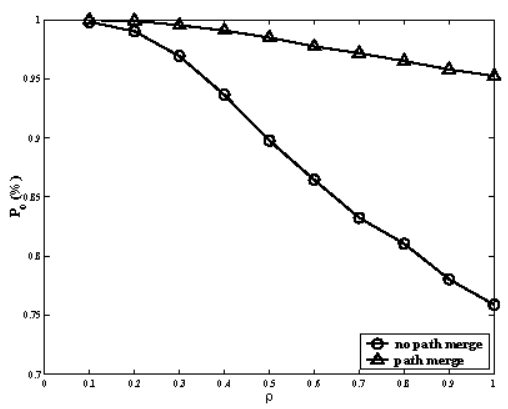

Figure 11: Impact of Path Merge mechanism on the optimized route reservation successful rate $P_{0}$.

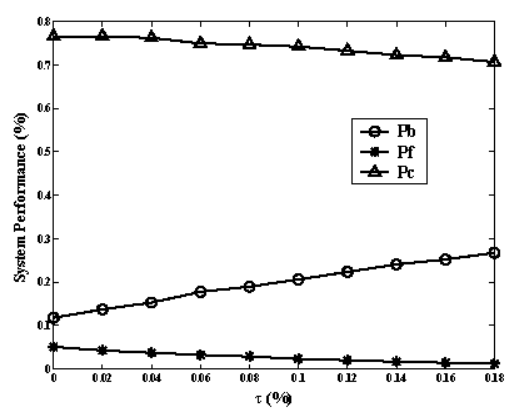

Figure 12: Impact of special reservation for handover sessions on the network performance indicators $P_{b}, P_{f}$ and $P_{c}$. 
hances the optimized route reservation successful rate Po (maintaining above 95\%). The reason is obvious; Path Merge avoids duplicate reservations, thus requiring fewer new resources when reserving on the optimized route. Besides, simulation results illustrated in Fig. 8 to Fig. 10 indicate that Path Merge also brings improvement in $P_{b}, P_{f}$ and $P_{c}$. This is because Path Merge enhances the optimized route reservation successful rate and this reduces resource wasting due to the triangular route. Thereby, the network performance is improved.

\subsection{Impact of Special Reservation for Handover Sessions on Network Per- formance}

In order to reduce the handover session forced termination rate, Fast RSVP introduces a new mechanism to reserve a part of network resources especially for handover sessions. In this subsection, we simulated and analyzed the impact of this mechanism on network performance. We define a new simulation parameter Handover Reservation Ratio $\zeta$ as the ratio of the amount of resources reserved especially for handover sessions to the total amount of network resources. The exact values of the simulation parameters are: $\rho=0.8, \lambda_{h}=100$ events $/ \mathrm{sec}, \mu_{h}=0.1 \mathrm{sec}^{-1}, \zeta=$ 00.2 . The simulation time is 1 day.

Fig. 12 depicts the variations of $P_{b}, P_{f}$ and $P_{c}$ as the Handover Reservation Ration $\zeta$ changes. It can be seen that with the increase of $\zeta$, handover session forced termination rate $P_{f}$ declines, accompanied by the rise of new session blocking rate $P_{b}$. The reason is apparent. Reserving part of network resources for handover sessions gives handover sessions a high priority thus making handover resource reservation requests more likely to be accepted. Meanwhile, the amount of resources left for new sessions decreases, making new sessions more likely to be rejected. It should be noted that in our simulation scenario the overall session completion rate $P_{c}$ declines as $\zeta$ gets larger. By observing the curve variation trends in Fig. 12, we can find that when $\zeta$ is small, $P_{f}$ declines faster and $P_{c}$ declines slower; but when $\zeta$ is large, $P_{f}$ declines slower and $P_{c}$ declines faster. Therefore, in real systems, network administrators should establish the proper amount of resources reserved for handover sessions according to the distribution of different kinds of sessions, so that the handover session forced termination rate is reduced while the new session blocking rate and overall session completion rate is not seriously deteriorated.

\section{Conclusion}

Aiming at supplying QoS guarantees to multimedia sessions, we proposed a new scheme, Fast RSVP, to reserve network resources in mobile IPv6 networks. The scheme, with the cooperation of the mobile IP and RSVP modules, can help a mobile node realize fast handover with QoS guarantees. Also, through the new mechanisms such as resource reservation on the optimized route and Path Merge, Fast RSVP avoids the resource wasting problem due to triangular routes and duplicate reservations. In addition, Fast RSVP introduces a new way to distinguish reservation requests from different types of sessions, giving handover sessions a higher priority and thus greatly reducing the handover session forced termination rate while maintaining high performance of the network.

\section{References}

[1] R. Braden and L. Zhang. Resource Reservation Protocol (RSVP) - version 1 functional specification. RFC2205, Sep 1997

[2] A. Terzis, M. Srivastava and L. Zhang. A Simple QoS Signaling Protocol for Mobile Hosts in the Integrated Services Internet. In Proc. of INFOCOM1999, NY, pp 1011-1018

[3] A. Terzis, J. Krawczyk, J. Wroclawski and L. Zhang. RSVP Operation over IP Tunnels. RFC2746, January 2000

[4] A. K. Talukdar, B. R. Badrinath and A. Acharya. MRSVP: A Resource Reservation Protocol for an Integrated Services Network with Mobile Hosts. In Wireless Networks Vol. 7 2001, pp 5-19

[5] W. Chen and L. Huang. RSVP Mobility Support: A Signaling Protocol for Integrated Services Internet with Mobile Hosts. In Proc. of INFOCOM2000, Tel Aviv, Israel, pp 1283-1292

[6] H. Jeon, M. Kim, K. Lee, J. Mo and D. Lee. Link Layer Assisted Multicast-Based Mobile RSVP(LM-MRSVP). In Proc. of ICOIN2005, Cheju Island, Korea, pp 452462

[7] V. Bharghavan and M. Jayanth. Profile-based Next-cell Prediction in Indoor Wireless LAN. In Proc. of IEEE SICON'97.

[8] J. Capka and R. Boutaba. Mobility Prediction in Wireless Networks Using Neural Networks. In Proc. of the 7 th International Conference on Management of Multimedia Networks and Services (MMNS2004), San Diego, California, USA, October 3-6, 2004.

[9] D. Johnson, C. Perkins and J. Arkko. Mobility Support in IPv6. RFC3775, June 2004 